\title{
A push-and-pull model for allosteric anion binding in cage complexes $\dagger$
}

\author{
Johannes M. Dieterich, ${ }^{a}$ Guido H. Clever ${ }^{b}$ and Ricardo A. Mata ${ }^{* a}$ \\ Received 30th May 2012, Accepted 17th July 2012 \\ DOI: $10.1039 / \mathrm{c} 2 \mathrm{cp} 41793 \mathrm{f}$
}

A series of electronic structure calculations has been carried out on an artificial anion binding host. The compound with four Pd(II) cations and a total of eight bis-monodentate pyridyl ligands forms by self-assembly an interpenetrated double cage with three binding pockets. Through the use of a simple pushand-pull model connecting the potentials of the different sites, we are able to explain the allosteric effect observed in anion binding. Two factors seem to be particularly significant in the latter, namely the flatness of the potential in each binding pocket as well as the length of the ligand. Our results are found to be in excellent agreement with the experimentally observed structures.

Anion binding is a widespread phenomenon in Nature due to the great importance of anions such as the halides, carboxylates and organic phosphates in biological systems. ${ }^{1}$ In particular chloride is an essential component of all body fluids and its defective regulation is associated with diseases such as cystic fibrosis and impairment of muscle function. ${ }^{2}$ A widespread feature of many biological systems (hosts) that are able to bind more than one charged or uncharged small molecule (guests) is cooperativity. ${ }^{3}$ Of particular interest are hosts that show positive homotropic cooperativity which, in connection with a concerted geometrical change of the host structure, is also termed allosteric binding. ${ }^{4}$ Since all individual binding sites of such a homotropic cooperative host are usually required to show the same local structure, a high symmetry of the overall structure is a prerequisite in these systems. One of the authors has recently reported an artificial halide anion binding host based on an interpenetrated double cage (DC) consisting of four $\mathrm{Pd}(\mathrm{II})$ cations and eight neutral bis-monodentate pyridyl ligands that forms quantitatively by a spontaneous selfassembly process in acetonitrile solution ${ }^{5}$ (see Fig. 1a). The design and rational synthesis of such interpenetrated cages and related knot structures ${ }^{6}$ are currently topics of great interest in supramolecular coordination chemistry. ${ }^{7,8}$ The single crystal X-ray structure showed that this dimeric double cage features three cavities, all filled initially with a tetrafluoroborate anion. Upon addition of two equivalents of halide anions per host, the two $\mathrm{BF}_{4}{ }^{-}$anions residing in the outer two pockets are quantitatively replaced by a halide anion. The anion exchange in these

\footnotetext{
${ }^{a}$ Institut für Physikalische Chemie, Universität Göttingen,

Tammannstr.6, 37077 Göttingen, Germany.E-mail: rmata@gwdg.de

${ }^{b}$ Institut für Anorganische Chemie, Universität Göttingen,

Tammannstr. 4, 37077 Göttingen, Germany

$\dagger$ Electronic supplementary information (ESI) available: Potential energy

fits, model structures in $x y z$ format. See DOI: $10.1039 / \mathrm{c} 2 \mathrm{cp} 41793 \mathrm{f}$
}

outer pockets is a fast process (in the order of $10^{3} \mathrm{~s}^{-1}$ at $298 \mathrm{~K}$ for $\mathrm{BF}_{4}{ }^{-}$in acetonitrile) as determined by NMR line width analysis and an EXSY (EXchange SpectroscopY) experiment. The $\mathrm{BF}_{4}^{-}$anion in the central pocket, however, does not exchange with the surrounding solution and cannot be replaced by the addition of halide anions. Addition of only one equivalent of halide anions leads to a $1: 1$ mixture of the unsubstituted host and the host with two halide anions bound. Among the halides, chloride is bound particularly strong by this homotropic positive cooperative binding mechanism accompanied by a compression of the double cage along the $\mathrm{Pd}_{4}$ axis, as indicated by a diffusion ordered spectroscopy (DOSY) NMR experiment. ${ }^{5}$ The possibility to dissolve $\mathrm{AgCl}$ in an acetonitrile solution of the double cage serves as strong evidence for an extraordinary affinity of this system for the binding of two chloride anions. To gain further insight into the factors responsible for the immense strength of the chloride binding and the magnitude of the structural change associated with the allosteric binding process, we conducted a computational study whose details and results are reported here.

There are several challenges to the theoretical description of this system. First of all, considering the double cage structure alone, one would be dealing with a total of 404 atoms. Such a system size would still be accessible to density functional methods, but requires significant computational resources. Furthermore, one is interested in obtaining insight into the energetic landscape, which would require extensive sampling. We have opted instead to build a simplified model for the system, which we hope not only reduces the computational cost of the study at hand, but also helps in better understanding the forces underlying its interesting properties.

The diagram shown in Fig. $1 \mathrm{~b}$ gives a schematic representation of the model used in this work. The double cage (DC) structure is reduced to four planes (Fig. 1c). Each plane consists of a $\operatorname{Pd}(\mathrm{II})$ cation coordinated to four pyridines (Pyr). An individual cage corresponds to two planes, constrained at a distance of $16.34 \AA$, the value found in the crystal structure with $\mathrm{BF}_{4}^{-}$anions. There are three positions within the boundaries of the double cage system which can be occupied by anions, which in turn define three subsystems. Each subsystem consists of two planes with an ion in the middle and is characterized by a distance $\left(r_{1}, r_{2}\right.$ or $\left.r_{3}\right)$. The distances are determined for a given ion configuration according to the sum of the three potentials

$$
V_{\mathrm{tot}}\left(r_{1}, r_{2}, r_{3}\right)=V_{1}\left(r_{1}\right)+V_{2}\left(r_{2}\right)+V_{3}\left(r_{3}\right)
$$




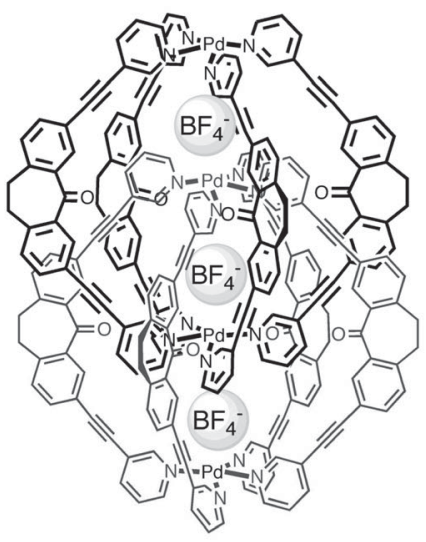

(a)

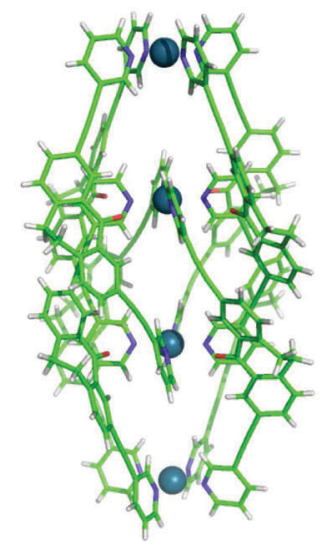

(b)

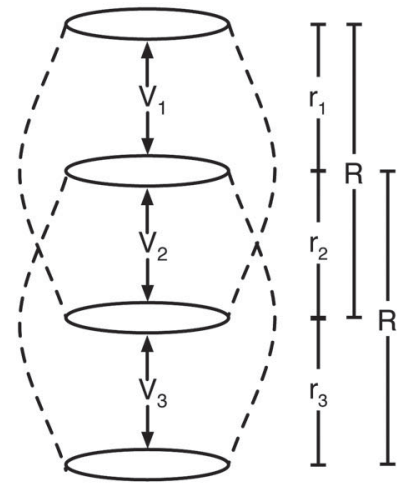

(c)

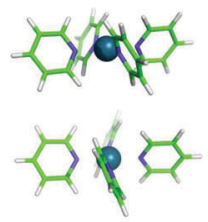

(d)

Fig. 1 (a) Molecular structure of the DC complex, showing the three binding sites with $\mathrm{BF}_{4}^{-}$; (b) 3-D representation of the complex crystal structure; (c) diagram representing the push-and-pull model used. The planes linked through the cage structure are kept at a fixed distance $R=$ $16.34 \AA$ A. A potential is associated with each binding pocket; (d) two $\left[\mathrm{Pd}(\mathrm{Pyr})_{4}\right]^{2+}$ complexes, which define two consecutive planes.

The $V_{x}$ terms correspond to the potential of a single anion contained between two consecutive planes (as shown in Fig. 1d). Since the planes in each cage are constrained to a distance $R$, the total potential of the system $V_{\text {tot }}$ only depends in fact on a single variable, which can be chosen to be the distance between the two planes in the middle.

$$
V_{\text {tot }}\left(r_{2}\right)=V_{1}\left(R-r_{2}\right)+V_{2}\left(r_{2}\right)+V_{3}\left(R-r_{2}\right) \text {. }
$$

Furthermore, potentials $V_{1}, V_{2}$ and $V_{3}$ are taken to depend solely on the anion which is found in between the two respective planes. For the study at hand, $V_{\text {tot }}$ can be built by using only two sets of potentials, one where a chloride anion is contained between the two planes, and another set for $\mathrm{BF}_{4}{ }^{-}$.

The individual potentials used in eqn (2) were computed as follows. The $\left[\mathrm{Pd}(\mathrm{Pyr})_{4}\right]^{2+}$ complex was first optimized at the BP86/def2-SVP level of theory. ${ }^{9-13}$ Afterwards, potential energy curves were computed for a model system including two $\left[\mathrm{Pd}(\mathrm{Pyr})_{4}\right]^{2+}$ complexes (planes) and an anion, $\mathrm{BF}_{4}{ }^{-}$or $\mathrm{Cl}^{-}$. In the case of $\mathrm{Cl}^{-}$, the plane geometries were kept parallel and at a fixed distance, with the anion placed in the middle of the axis defined by the two Pd atoms. One of the complexes was rotated $20^{\circ}$ relative to the other plane, in agreement with the averaged angles in the crystal structure of $\left(\mathrm{BF}_{4}^{-}\right)_{3} @ \mathrm{DC} .^{5}$ In the case of $\mathrm{BF}_{4}^{-}$, BP86/def2-SVP geometry optimizations were carried out, but only relaxing the anion atoms. The planes internal geometry as well as the distance were kept fixed at each point. The final potential energy curves were obtained with single point DF-MP2/cc-pVTZ (cc-pVTZ-PP for Pd, in combination with the ECP28MDF pseudopotential) calculations. ${ }^{14,15}$ All DFT calculations were carried out with the Orca program package, ${ }^{16}$ the MP2 calculations with Molpro2010.1. ${ }^{17}$

In order to effectively use eqn (2), the data points were fitted to a sum of gaussian functions of the form

$$
V_{A}=\sum_{i}^{N_{\mathrm{G}}} A_{i} \mathrm{e}^{-\alpha_{i}\left(r_{A}-\lambda\right)^{2}}
$$

where $r$ is the distance in between the planes and $A_{i}, \alpha_{i}$ and $\lambda_{i}$ are free parameters in the global fit. Five Gaussians were used to obtain a converged description of the potential. The global parametrization was based on genetic algorithms as implemented in the OGOLEM framework for global optimization ${ }^{18-20}$ using a pool of 1000 individuals and 99000 global optimization steps with local optimizations. Standard single-point genotype crossover and mutation operators were used. An energy diversity of $1 \times 10^{-5} E_{\mathrm{h}}$ was employed to avoid premature convergence of the genetic pool. Further information can be found in the ESI. $\dagger$

Before we discuss any results, the approximations in the model used should be shortly revised. The two major underlying assumptions are:

- the cage monomer geometry does not change, independent of the anion species present, or at least should not have a significant effect in the overall process.

- the only dominant interactions which depend on the anion are between the latter and the $\left[\mathrm{Pd}(\mathrm{Pyr})_{4}\right]^{2+}$ planes.

The first assumption is perhaps the most debatable. The monomers, even if somewhat hindered by the interlocking, should be able to slightly change their geometry. The ligands themselves contain two major degrees of freedom that can lead to some degree of flexibility. One is the rotation around the alkyne functionality. In the context of the cage assembly this is directly linked to the propeller twist of the $\left[\mathrm{Pd}(\mathrm{Pyr})_{4}\right]^{2+}$ planes internal geometry, which is fixed throughout this study. A second element of flexibility may be attributed to the conformation of the seven-membered ring of the ligands dibenzosuberone backbone. According to the X-ray structure of the double cage, all eight ligand backbones adopt a $C_{2}$-symmetric twist conformation with the twofold axis cutting through the middle of the ethylene bridge and being collinear with the carbonyl groups $\mathrm{C}=\mathrm{O}$ bond. ${ }^{1} \mathrm{H}$ NMR data, however, indicate that a ringflip of the seven membered ring is only possible for the free ligand, not for the ligand after it has been incorporated inside the densely packed double cage assembly. Regarding the overall helical structure of the interpenetrated cages, we further expect another motion to occur concomitantly with the chloride-induced compression along the $\mathrm{Pd}_{4}$ axis. Since the cages are entangled in a screw-like arrangement, any linear movement along this line should be accompanied by 


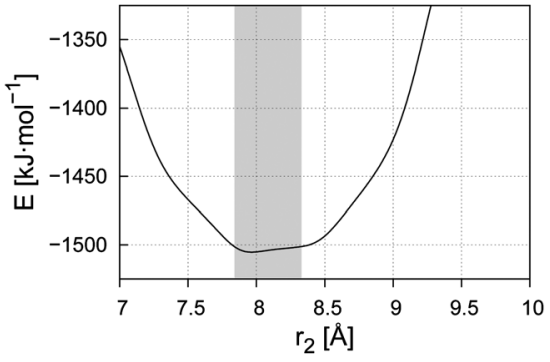

(a)

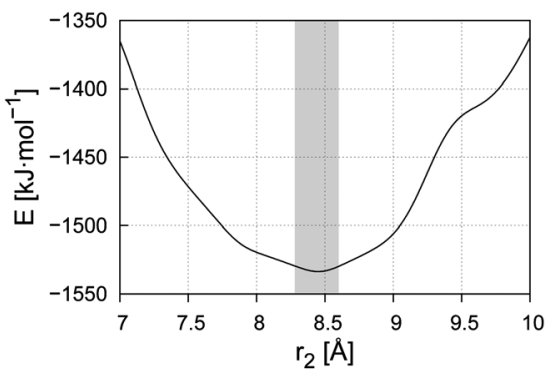

(b)

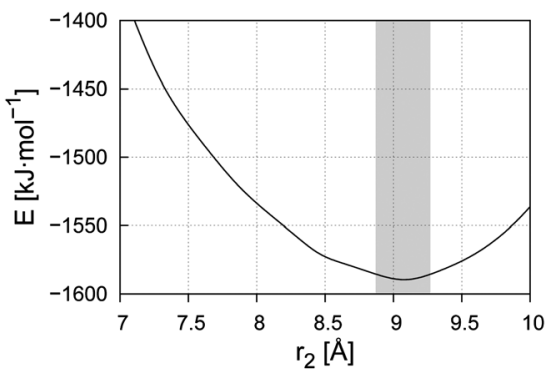

(c)

Fig. 2 Potential energy curves for (a) $\left(\mathrm{BF}_{4}^{-}\right)_{3} @ \mathrm{DC}$, (b) $\left(\mathrm{BF}_{4}^{-}\right)_{2} \mathrm{Cl}^{-} @ \mathrm{DC}$ and (c) $\left(\mathrm{BF}_{4}^{-}\right)\left(\mathrm{Cl}^{-}\right)_{2} @ \mathrm{DC}$. The energies were obtained according to eqn (2). The planes linked through the cage structure are kept at a fixed distance $R=16.34 \AA$.

a slight rotation of the two cages with respect to each other (screw motion). Since such a rotation should not significantly affect the distance dependent interaction between the $\left[\mathrm{Pd}(\mathrm{Pyr})_{4}\right]^{2+}$ planes and the anion sandwiched between them, we chose to neglect any rotational movement in our current model.

The second approximation is not as severe. There are no strong interaction points on the side arms which could differentiate significantly between the two anions. Possible contributions should be of the same order of magnitude as other neglected effects, such as outer solvation or nonadditivity in the coordination to the Pd-centers.

The computed potentials are shown in Fig. 2. They depict three different binding combinations, considering single and double substitution of $\mathrm{BF}_{4}^{-}$by $\mathrm{Cl}^{-}$in the DC system:

$$
\text { Step 1: }\left(\mathrm{BF}_{4}^{-}\right)_{3} @ \mathrm{DC} \rightarrow\left(\mathrm{BF}_{4}{ }^{-}\right)_{2} \mathrm{Cl}^{-} @ \mathrm{DC}
$$

\section{Step 2: $\left(\mathrm{BF}_{4}^{-}\right)_{2} \mathrm{Cl}^{-} @ \mathrm{DC} \rightarrow \mathrm{BF}_{4}^{-}\left(\mathrm{Cl}^{-}\right)_{2} @ \mathrm{DC}$}

As mentioned previously, substitution only takes place in the outer pockets. Information about the structural minima and energies is compiled in Table 1.

We start by discussing the results for the $\left(\mathrm{BF}_{4}{ }^{-}\right)_{3} @ \mathrm{DC}$ case. The potential curve displays, rather surprisingly, a broad minimum at around $r=7.96 \AA$. This value should, however, be considered with caution, since the distance range between 7.84 and $8.33 \AA$ is within $1 \mathrm{kcal} \mathrm{mol}^{-1}$ or less. Large amplitude motions should be expected, which reduces the significance of using a single distance. The range $8.09 \pm 0.25 \AA$ is best suited to describe the theoretical estimate (with a $1 \mathrm{kcal} \mathrm{mol}^{-1}$ error barrier). The crystal structure of $\left(\mathrm{BF}_{4}^{-}\right)_{3} @ \mathrm{DC}$ yielded values of 8.093(3) and 8.251(3) $\AA$ for the distance between the Pd(II) cations defining the outer and inner cavities, respectively. These experimental values are in good agreement with our theoretical results.

Table 1 Computed values for the minima wells for different anion bindings in the DC complex (interplanar distance in $\AA$ and lowest interaction energies in $\mathrm{kJ} \mathrm{mol}^{-1}$ )

\begin{tabular}{llll}
\hline Anions & $r_{2}$ (calc.) & $r_{2}(\exp )$ & $V_{\text {tot }}$ \\
\hline $\mathrm{BF}_{4}^{-} / \mathrm{BF}_{4}^{-} / \mathrm{BF}_{4}^{-}$ & $8.09 \pm 0.25$ & 8.251 & -1505.3 \\
$\mathrm{Cl}^{-} / \mathrm{BF}_{4}^{-} / \mathrm{BF}_{4}^{-}$ & $8.44 \pm 0.16$ & - & -1533.6 \\
$\mathrm{Cl}^{-} / \mathrm{BF}_{4}^{-} / \mathrm{Cl}^{-}$ & $9.07 \pm 0.20$ & - & -1589.5 \\
\hline
\end{tabular}

Upon replacement of a single $\mathrm{BF}_{4}{ }^{-}$in one of the outer pockets by a chloride anion, there is a significant change in the potential. Comparing Fig. (2a) and (b), the minimum is shifted by $0.4-0.5 \AA$, leading to an effective contraction of the double cage structure (when the middle pocket expands, both outer sites contract). The $\mathrm{BF}_{4}{ }^{-}$binding energy in the opposite side of the cage is only slightly affected. The optimal minimum for a single $\mathrm{BF}_{4}{ }^{-}$in between two planes is found at $8.2 \AA$ (see ESI $\dagger)$. In the $\left(\mathrm{BF}_{4}{ }^{-}\right)_{2} \mathrm{Cl}^{-} @ \mathrm{DC}$ system, the outer $\mathrm{BF}_{4}{ }^{-}$ is contained between planes at $7.90 \pm 0.16 \AA$, which is at most $4 \mathrm{~kJ} \mathrm{~mol}^{-1}$ higher in energy from the optimal distance.

Finally, we examine the profile for the $\left(\mathrm{BF}_{4}{ }^{-}\right)\left(\mathrm{Cl}^{-}\right)_{2} @ \mathrm{DC}$ system. This shows a minimum at $9.07 \pm 0.20 \AA$, which in turn would mean an interplanar distance of $7.27 \pm 0.20 \AA$ for the outer sites. The middle site should be significantly stretched, contracting the overall structure. This leads to a more favorable binding of both outer chloride anions. Comparing the total interaction energy for each of the cases under study, one finds that the first substitution (Step 1) leads to a lowering in the energy of $28.3 \mathrm{~kJ} \mathrm{~mol}^{-1}$. The second substitution (Step 2), on the other hand, is favored by $55.9 \mathrm{~kJ} \mathrm{~mol}^{-1}$ (a total of $84.2 \mathrm{~kJ} \mathrm{~mol}^{-1}$ for completely replacing the outer anions). This is a clear indication of the allosteric effect. There is a much more significant energy gain replacing both sites than binding a single chloride. One determining factor for the allosteric effect to take place is the form of the $\mathrm{BF}_{4}{ }^{-}$potential. Expanding the inner pocket by about $1 \AA$ does not lead to any severe energy penalty.

According to our model, we would expect total structure sizes (distance between the outer $\mathrm{Pd}$ (II) centers) of 24.59, 24.24 and $23.61 \AA$ for $\left(\mathrm{BF}_{4}^{-}\right)_{3} @ \mathrm{DC},\left(\mathrm{BF}_{4}^{-}\right)_{2} \mathrm{Cl}^{-} @ \mathrm{DC}$ and $\left(\mathrm{BF}_{4}^{-}\right)\left(\mathrm{Cl}^{-}\right)_{2} @ \mathrm{DC}$ respectively. This would indicate a total axis contraction of about $4 \%$, from the first to the doubly substituted system. A comparison to the experimental value for the extent of chloride-induced shrinking that was previously obtained by DOSY NMR is worth considering. The DOSY values hint at a $4 \%$ overall shrinkage in radius. This has been roughly calculated from the measured differences in the diffusion coefficients. ${ }^{5}$ The exact relation between the radius in the Stokes-Einstein equation and the main axis length is uncertain, since it depends on the shape of the double cage and how anisotropy is handled. A $4 \%$ to $12 \%$ compression along the main axis would be expected. Smaller compressions seem to be more plausible, based on a space filling model 
of the double cage. Whereas such total distance values extracted from a DOSY experiment are known to suffer from quite large errors (changes in the ionic strength as well as the counteranion and solvation influences are not considered at all), both the DOSY values and the calculations described here are in reasonable agreement and clearly support the anticipated compression along the $\mathrm{Pd}_{4}$ axis upon binding of two chloride anions.

In conclusion, the calculated results are in full agreement with the experimental observations of chloride binding in the outer two pockets of the interpenetrated double cage with positive cooperativity. The structural change (compression along the $\mathrm{Pd}_{4}$-axis) associated with the anion binding process is convincingly reproduced. Our calculations thus provide a solid basis for the understanding of allosteric anion binding processes in interpenetrated coordination cages of the type $\left[\mathrm{Pd}_{4} \mathrm{~L}_{8}\right]$ which greatly facilitates the rational design of a next generation of modified systems with tunable anion binding affinity and selectivity. According to our model, the cage complexes could be effectively modified by changing the anion in the central pocket and varying the ligand to shorten/expand the cage. Depending on the interplay of these factors, the allosteric effect could be designed for a specific class of anions.

\section{Acknowledgements}

Financial support from the German Excellence Initiative, through the Free Floater Research Group program of the University of Göttingen, is gratefully acknowledged. R. A. M. and G. H. C. also acknowledge support from the International Research Training Group 1422.

\section{References}

1 J. L. Sessler, P. Gale, W.-S. Cho and S. J. Rowan, Anion Receptor Chemistry (Monographs in Supramolecular Chemistry), Royal Society of Chemistry, 2006.

2 R. Dutzler, E. B. Campbell, M. Cadene, B. T. Chait and R. MacKinnon, Nature, 2002, 415, 287.

3 J.-P. Changeux and S. J. Edelstein, Science, 2005, 308, 1424.

4 J. Monod, J. Wyman and J.-P. Changeux, J. Mol. Biol., 1965, $\mathbf{1 2}, 88$.

5 S. Freye, J. Hey, A. T. Galán, D. Stalke, R. H. Irmer, M. John and G. H. Clever, Angew. Chem., Int. Ed., 2012, 51, 2191.

6 D. M. Engelhard, S. Freye, K. Grohe, M. John and G. H. Clever, Angew. Chem., Int. Ed., 2012, 51, 4747.

7 R. S. Forgan, J.-P. Sauvage and J. F. Stoddart, Chem. Rev., 2011, 111, 5434 .

8 J. E. Beves, B. A. Blight, C. J. Campbell, D. A. Leigh and R. T. McBurney, Angew. Chem., Int. Ed., 2011, 50, 9260.

9 A. D. Becke, Phys. Rev. A: At., Mol., Opt. Phys., 1988, 38, 3098.

10 J. P. Perdew, Phys. Rev. B: Condens. Matter Mater. Phys., 1986, 33, 8822.

11 A. Schaefer, H. Horn and R. Ahlrichs, J. Chem. Phys., 1992, 97, 2571.

12 F. Weigend and R. Ahlrichs, Phys. Chem. Chem. Phys., 2005, 7, 3297.

13 D. Andrae, U. Haeussermann, M. Dolg, H. Stoll and H. Preuss, Theor. Chim. Acta, 1990, 77, 123.

14 T. H. Dunning, J. Chem. Phys., 1989, 90, 1007.

15 K. Peterson, D. Figgen, M. Dolg and H. Stoll, J. Chem. Phys., 2007, 126, 124101.

16 F. Neese, Orca: An Ab Initio, DFT and Semiempirical electronic structure package v2.8, 2011.

17 H.-J. Werner, P. J. Knowles, G. Knizia, F. R. Manby and M. Schütz, et al., MOLPRO, version 2010.1, a package of ab initio programs, 2010, see http://www.molpro.net.

18 J. M. Dieterich and B. Hartke, Mol. Phys., 2010, 108, 279.

19 J. M. Dieterich and B. Hartke, J. Comput. Chem., 2011, 32, 1377.

20 J. M. Dieterich, S. Gerke and R. A. Mata, J. At., Mol., Opt. Phys., 2012, 2012, 648386. 